\title{
Médiévales
}

Langues, Textes, Histoire

46 | printemps 2004

Éthique et pratiques médicales

\section{La rationalité médicale à l'épreuve de la peste : médecine, astrologie et magie (1348-1500)}

The medical rationality faced with the plague : medicine, astrology and magic (1348-1500)

\section{Nicolas Weill-Parot}

\section{(2) OpenEdition}

\section{Journals}

Édition électronique

URL : https://journals.openedition.org/medievales/884

DOI : $10.4000 /$ medievales.884

ISSN : $1777-5892$

Éditeur

Presses universitaires de Vincennes

Édition imprimée

Date de publication : 1 juin 2004

Pagination : 73-88

ISBN : 2-84292-151-8

ISSN : 0751-2708

\section{Référence électronique}

Nicolas Weill-Parot, « La rationalité médicale à l'épreuve de la peste : médecine, astrologie et magie (1348-1500) », Médiévales [En ligne], 46 | printemps 2004, mis en ligne le 02 mars 2006, consulté le 22 avril 2022. URL : http://journals.openedition.org/medievales/884; DOI : https://doi.org/10.4000/ medievales.884

Ce document a été généré automatiquement le 22 avril 2022.

Tous droits réservés 


\section{La rationalité médicale à l'épreuve de la peste : médecine, astrologie et magie (1348-1500)}

The medical rationality faced with the plague : medicine, astrology and magic

(1348-1500)

Nicolas Weill-Parot

1 La pensée scolastique dans sa volonté d'explorer tous les possibles et d'en proposer une explication rationnelle exprime une forme de confiance dans la puissance de la raison à rendre compte de tout le donné créé. La médecine scolastique-c'est-à-dire la médecine savante - se construit à partir du XII ${ }^{\mathrm{e}}$ siècle sur une autonomie structurelle affirmée et une rationalité assez rigoureuse. Il n'y a plus guère de place pour les charmes qui étaient courants dans la médecine de recettes du haut Moyen Âge, et encore moins pour la magie savante ou demi-savante véhiculée par des textes traduits de l'arabe, et peut-être aussi du grec, à partir du $\mathrm{XII}^{\mathrm{e}}$ siècle $^{1}$. Les formes de magie comme la magie astrale hermétique, la magie rituelle salomonienne ou la "nicromancie» n'avaient évidemment aucune chance de se retrouver dans la médecine enseignée dans les universités du XIII ${ }^{\mathrm{e}}$ siècle et de la première moitié du XIV siècle.

Le problème de l'astrologie ne se pose pas tout à fait dans les mêmes termes: la rationalité des règles astrologiques permet des associations plus cohérentes que la magie avec la médecine savante. Pourtant, les travaux de Danielle Jacquart conduisent à minimiser la place de la science des astres dans la médecine universitaire ${ }^{2}$. Le médecin ordinaire, formé à l'université, ne se servait généralement de pronostics astrologiques que dans un nombre limité de cas, notamment pour la saignée. Bien sûr, certains médecins sont allés beaucoup plus loin dans l'utilisation de l'astrologie - et ce ne sont pas les moins célèbres, puisque l'on peut citer Pietro d'Abano († 1315/1316) ${ }^{3}$. Cependant la médecine scolastique ordinaire ne s'est guère servie des procédés astrologiques; ces derniers, en effet, risquaient de court-circuiter la logique propre à la 
méthode médicale de diagnostic et de thérapeutique, c'est-à-dire la rationalité propre à la médecine scolastique.

3 Mais lorsqu'en 1348 la peste déferla sur l'Europe, les médecins savants furent confrontés à un fléau contre lequel l'impuissance ou l'insuffisance de leur art apparaissait de façon évidente ${ }^{4}$. En d'autres termes, un défi surgissant du côté de la pratique mettait à l'épreuve la pertinence de l'édifice théorique de leur science. La question est donc de savoir s'ils ouvrirent grand les digues qui séparaient leur discipline du monde plus ou moins trouble des arts marginaux ou occultes. La peste sonna-t-elle le glas de la rationalité auto-suffisante de la médecine scolastique médiévale, sous le coup de la découverte trop flagrante de son inadéquation à la pratique?

4 De façon générale, la peste du bas Moyen Âge appelle une série d'images, d'idées et de scènes qui évoquent une fuite échevelée vers l'irrationnel. Au-delà du conseil (qui n'est pas si déraisonnable d'un point de vue individuel...) de fuir le plus loin les lieux infectés par le fléau, viennent en mémoire les massacres des juifs accusés d'être les fauteurs de l'épidémie, les processions de flagellants ou l'essor concomitant d'une théâtralisation du macabre ${ }^{5} .$. La médecine fut-elle affectée par ces phénomènes ? La peste aurait-elle contribué à ébranler l'édifice conceptuel de cette médecine rationnelle, laissant par les brèches s'engouffrer les éléments plus ou moins magiques appelés à répondre de façon pitoyable aux inquiétudes mortelles des contemporains?

5 Chiara Crisciani et Michela Pereira ont mis en évidence les éventuelles inflexions alchimiques du discours médical confronté à l'épidémie de peste. Elles ont repéré, par exemple, l'utilisation de l'or dans certains écrits sur la peste. Inversement, les alchimistes se sont aussi préoccupés de trouver un remède contre le fléau. De ce point de vue, le parallèle dressé entre la pierre alchimique (le lapis) et la thériaque, mélange pharmacologique censé contrer tous les poisons, a été relevé6.

6 Du reste, les relations entre les «écrits sur la peste »-Pestschriften, pour reprendre la dénomination générale donnée par Karl Sudhoff ${ }^{7}$ - et la pratique sont aussi diverses que le genre littéraire dont relève chacun d'entre eux dans leur diversité : Regimen, Consilium, Tractatus ou Quaestio. Quoi qu'il en soit, la question de la pratique médicale en temps de peste et celle de la présence de l'astrologie et des sciences occultes dans la médecine savante se rencontrent dans deux registres de faits.

7 En premier lieu, l'existence même de la peste constitue un extraordinaire défi pratique pour la médecine savante. Les médecins se trouvent devant une situation d'urgence. Ils doivent y faire face alors même que la réalité pratique ne cesse d'apporter des démentis à leur système scientifique. Dans ces conditions, la première question consiste à se demander si c'est en rupture avec la rationalité médicale que l'explication astrologique fait son entrée remarquée dans l'étiologie proposée pour la peste.

8 En second lieu, dans les solutions pratiques à apporter au fléau, les médecins ont dû recourir à un certain nombre de procédés prophylactiques et thérapeutiques. Compte tenu de l'inadéquation entre leur système scientifique et la réalité qui s'imposait à eux implacablement, ont-ils eu recours massivement à des procédés magiques et magicoastrologiques? Et ce recours trahit-il chez eux une fuite vers l'irrationnel ou, du moins, à l'extérieur des clairs chemins de la médecine scolastique?

L'étiologie astrologique 
9 Le fait est bien connu : l'astrologie fut utilisée dans l'étiologie de l'épidémie de façon fréquente, quoique avec de sérieuses nuances entre les auteurs comme l'a souligné Jon Arrizabalaga, qui a rappelé également l'influence du Canon d'Avicenne où étaient distinguées les causes éloignées astrales et les causes proches (les dispositions terrestres) pour rendre compte de la putréfaction de l'air responsable du fléau ${ }^{8}$. Les traités pouvaient s'appuyer sur le célèbre avis rendu à la demande du roi Philippe VI par les médecins de l'Université de Paris en octobre 1348. En exploitant un passage d'Albert le Grand, les médecins parisiens avaient conclu que la conjonction des trois planètes supérieures (Saturne, Jupiter et Mars) dans le signe du Verseau en 1345 était responsable de la corruption de l'air, cause de la Peste noire de $1348^{\circ}$. Quelques mois plus tôt (en avril), en s'appuyant sur le même passage d'Albert le Grand, le catalan Jacme d'Agramont dans son Regiment de preservació a pestilència avait déjà écrit d'un point de vue seulement théorique qu'une conjonction Saturne - Jupiter dans le même signe et degré annonçait des mortalités ; mais, il est vrai, il n'insistait guère sur cette composante astrologique ${ }^{10}$.

10 La mise en avant d'une cause astrologique dans l'étiologie de la peste traduit-elle un bouleversement des règles scientifiques de la médecine savante? Une analyse précise permet, me semble-t-il, de répondre négativement. Le prologue de la consultation parisienne de 1348 frappe par le souci que manifestent les maîtres de se servir le plus rigoureusement possible des ressources de la raison scientifique et médicale. Ils expliquent que devant les phénomènes dont la cause échappe même aux plus ingénieuses des intelligences, l'homme est saisi d'étonnement, et que son désir inné de trouver le bien et le vrai le pousse à chercher les causes des effets merveilleux. Ils remarquent que c'est bien ce qu'ont fait les médecins et les astrologues. Mais comme l'analyse de ces derniers posait un certain nombre de problèmes, ils ont décidé, rappellent-ils, à la demande du roi, en s'aidant des propos des anciens et des modernes, des médecins et des astrologues, de faire le point sur les causes universelles et lointaines ainsi que sur les causes proches de l'épidémie et sur les remèdes à apporter " autant qu'il est dans la nature de ce problème de se soumettre à l'intellect humain » (quantum ipsius rei natura humano intellectui se subjicit) ${ }^{11}$. Au-delà de l'aspect rhétorique du prologue, la volonté d'expliquer est très nettement mise en avant, non seulement pour ne pas décevoir le roi de France qui a demandé cet avis, mais aussi parce que la méthode scolastique impose et suppose cette puissante volonté d'expliquer.

11 Dans la suite de la consultation, la constellation de 1345 est présentée comme une remota causa, une cause lointaine de la peste: Jupiter, planète chaude et humide, a soulevé depuis la terre l'eau et les vapeurs nocives, qui ont été ensuite embrasées par Mars, planète chaude et sèche. Or entre le 6 octobre 1347 et la fin de l'année 1348 se réunirent dans le signe du Lion Mars et la Tête du Dragon ${ }^{12}$; cette concentration d'entités astrales chaudes, en attirant en grand nombre les vapeurs, ont engendré un hiver trop chaud. De surcroit, Mars, parce qu'il était rétrograde, a attiré depuis la terre et l'eau de nombreuses vapeurs qui ont entraîné une corruption de l'air ${ }^{13}$.

Cette mise en avant d'une cause astrologique reste à plus d'un titre dans les limites du cadre naturaliste médical. En premier lieu, en effet, cette influence est censée n'avoir agi que sur un élément physique : l'air. Cette influence astrale entre donc dans la même catégorie que les phénomènes physiques (comme le mouvement des marées commandé par la lune) ou météorologiques (les variations atmosphériques) ${ }^{14}$. En second lieu, la cause astrologique se trouve intégrée sans difficulté à l'étiologie médicale alors en 
vigueur. En effet, l'influence de la constellation des trois planètes supérieures n'est pas considérée comme une cause immédiate et mystérieuse du mal; au contraire, son action a été médiatisée par toute une série de causes physiques qui entrent pleinement dans l'explication médicale du phénomène ${ }^{15}$. Dans la seconde moitié $d u$ XIv $^{e}$ siècle, le médecin Johann von Sachsen écrit dans son Compendium de epydemia : «Dieu et le ciel ne sont pas par eux-mêmes la cause de l'épidémie ». En ce qui concerne la cause céleste, le fait est évident, puisque la peste n'a pas atteint à tous les moments également toutes les régions ni tous les hommes. Ainsi, après avoir cité Avicenne, le médecin range les causes loitaines par ordre d'éloignement décroissant ainsi : Dieu, le ciel astral, puis l'air; et les causes proches par proximité croissante comme suit: l'humeur, l'air corrompu et la vapeur corrompue qui s'est introduite dans le cœur ${ }^{16}$. Un autre médecin, Primus de Corpusllirio (ou de Gorllicio), maître en médecine diplomé de l'Université de Paris, écrit au Xve siècle, dans son Brevis tractatus contra pestem :

De nombreux savants n'ont su attribuer la cause de cette terreur pestilentielle qu'à Dieu seul, cependant il est probable qu'elle est causée immédiatement par la constellation ou bien qu'elle l'est par le ciel au moyen de la vapeur corrompue, de même que le rayon est causé par le soleil; et cela semble être la thèse des astrologues qui disent qu'une planète est bienveillante et une autre malveillante ; mais un plus grand nombre de médecins ne prend pas cela en considération ${ }^{17}$.

Si Primus n'exclut pas a priori la possibilité d'une influence immédiate des astres, il présente en tout cas la causalité astrologique en elle-même comme une explication scientifique qui évite le recours affolé à l'explication par le châtiment divin.

On pourrait cependant se demander si une étiologie qui faisait la part belle à une cause lointaine et hors de portée, comme l'est une conjonction de planètes, n'a pas pu jouer un rôle démobilisateur dans le désir de contrer le fléau, conduisant ainsi les hommes par désespoir vers les refuges les plus irraisonnés. Il est vrai qu'au tout début de son Consilium ad pestilentiam, Giovanni Santasofia (mort en 1389) écrit : "... cette peste si étonnante et si horrible procède de causes supérieures qui régissent et gouvernent nos corps et dont nous ne pouvons empêcher les influences $»^{18}$. Cependant ce constat sur l'impossibilité de contrer des influx astraux ne signifie pas un renoncement à lutter contre l'effet lui-même: la corruption de l'air et la maladie qui s'ensuit; et, au demeurant, le même Giovanni di Santa Sofia poursuit :

Parce que cependant, selon Galien, dans le premier livre des fièvres, la plus grande partie de la génération des maladies est indistinctement la préparation du corps, je présenterai par écrit quelques remèdes approuvés par nos auteurs et éprouvés lors d'autres pestes qui, même s'ils n'empêchent pas les influences des corps supérieurs, préparent nos corps de façon à ce qu'ils ne subissent pas facilement les causes supérieures indiquées plus haut ${ }^{19}$.

La cause astrologique n'a donc pas démobilisé l'esprit rationalisant des médecins universitaires. Cette rationalisation est une caractéristique de l'esprit scolastique médiéval, qui recherche le plus loin qu'il peut les moyens d'encadrer le donné, même le plus apparemment inexplicable.

16 Il est possible qu'au $\mathrm{XV}^{\mathrm{e}}$ siècle on assiste cependant à une inflexion de la causalité astrologique dans l'analyse de la peste. Les prédispositions astrologiques de certains individus ou de certains lieux sont envisagés dans quelques écrits ${ }^{20}$. Dès lors, l'étiologie médicale risque d'être concurrencée par ce type de causalité. L'exemple de l'étrange justification astrologique d'une rumeur anti-juive peut cependant nuancer l'évaluation globale de cette perturbation de la rationalité médicale. Les médecins furent en effet 
confrontés à une croyance populaire qui faisait des juifs les fauteurs de l'épidémie. Comme on le sait, le pape Clément VI condamna ces rumeurs en faisant remarquer notamment que la peste frappait autant les juifs que les chrétiens ${ }^{21}$. Le chirurgien Guy de Chauliac et quelques autres savants s'élevèrent ouvertement contre l'idée que les juifs fussent plus épargnés que les chrétiens ${ }^{22}$. Pourtant cette croyance, qui en était restée au milieu du XIV siècle à un niveau populaire assez bas, réapparut au Xve siècle sous la plume du médecin Primus de Corpusllirio ; toutefois, l'explication qu'il donne essaie de rationaliser «scientifiquement » et astrologiquement ce prétendu fait, et ne verse pas dans la théorie du complot à l'origine des massacres des années 1348-1350 :

Le troisième problème est que les juifs meurent moins que les chrétiens. Il est répondu que, en raison du mode de vie qui leur est propre, <les juifs> ne s'exposent pas à l'air du matin et du soir et qu'ils ne se promènent pas ainsi à l'air extérieur comme les chrétiens; or cet air extérieur est plus mauvais en temps de peste, lorsque les astres ont de mauvaises influences. On peut dire que les juifs sont saturniens, et que la complexion de leur planète est froide et sèche, <c'est-à-dire> opposée à la maladie pestilentielle, et qu'ainsi ils résistent davantage et sont moins exposés à cet air infecté ; et c'est ainsi que les juifs meurent moins ${ }^{23}$.

$A u$-delà du problème que pose cette résurgence d'une croyance anti-judaïque ${ }^{24}$, dans la perspective de la présente étude, l'astrologie apparait ici comme un outil permettant de rationaliser de façon naturaliste et médico-astrologique toutes sortes d'idées, même les idées les plus folles. Cette justification, qui rejoint l'étiologie de la peste (la corruption de l'air) et la théorie des prédispositions (la complexion des corps), ne paraitt pas perturber en dernier ressort la rationalité médicale alors en vigueur.

Une première conclusion s'impose donc sur la nature des liens noués par les médecins avec l'astrologie dans leurs écrits sur la peste. Confrontés à un problème pratique de grande ampleur - la grande mortalité qui défiait le cadre normal de la médecine -, les médecins n'ont pas quitté leur propre grille d'explication rationnelle; à un fléau énorme ils ont donné une cause énorme : la conjonction des planètes supérieures. Mais ce type d'explication n'a généralement pas bouleversé leur étiologie médicale ordinaire : les astres firent simplement figures de cause lointaine. Elle ne les a pas non plus conduits à baisser les bras devant l'impossibilité de lutter contre une cause si puissante et hors d'atteinte. En somme, le recours à l'astrologie face à la peste n'a détruit ni l'étiologie médicale rationnelle ni la fonction pratique de la médecine.

Les écrits sur la peste ne se contentent pas de livrer une étiologie, ils exposent aussi une prophylaxie et une thérapeutique. Il s'agit donc de déterminer dans quelle mesure ces domaines ont été propices aux infiltrations magico-astrologiques.

La magie et le recours aux empirica

Dans les prescriptions que livrent ces écrits, trouve-t-on des éléments magiques ? Il est tout d'abord évident qu'on ne peut trouver des rituels « nicromantiques » que l'Église aurait immédiatement condamnés dans ces traités écrits par des médecins savants, dédiés très souvent à de hauts personnages et destinés à une large diffusion. On pourrait, en revanche, s'attendre à trouver là (si l'on adopte l'hypothèse d'un effondrement de l'édifice théorique médical) des traces de magie para-religieuse, c'està-dire des utilisations à moitié superstitieuses de prières, à la façon des charmes de la magie monastique et populaire du haut Moyen Âge, comme on en trouvait encore des traces dans certains cas d'école comme l'épilepsie (ce qui n'est évidemment pas la même chose que la magie savante théurgique ou angélique, franchement hétérodoxe) ${ }^{25}$. Ce ne semble pas être le cas, ce qui renvoie, au demeurant, à un autre trait distinctif du 
discours médical : la part relativement restreinte faite à la notion de châtiment divin (à moins que la cause divine ne soit elle-même intégrée à l'étiologie scientifique comme cause lointaine, ce qui la neutralise).

$\mathrm{Au}$ milieu des conseils prophylactiques souvent ordonnés suivant les «six choses non naturelles ", et les prescriptions thérapeutiques, il faut donc guetter des formes de magie, pour ainsi dire, moins explicitement magique: c'est-à-dire les procédés empiriques, les empirica, ayant une part de merveilleux. Dans un contexte d'appel d'urgence venu de la pratique et peut-être d'inadéquation patente entre les procédés méthodologiquement déductibles des principes galéniques et la présence subite et implacable du fléau, le recours aux empirica a pu apparaître comme une voie particulièrement tentante. Mais, là encore, il faut cerner de près la nature de ces empirica. Depuis ses origines, en effet, la médecine scolastique avait intégré toute une série de médicaments empiriques dont la vertu ne pouvait être connue que par l'expérience (et donc par la tradition) et non par la doctrine galénique des complexions (agencement des qualités froide, chaude, sèche et humide). Pour ce faire, les médecins disposaient d'une notion susceptible d'intégrer l'inexpliqué à une explication rationnelle : la notion de forme spécifique. Celle-ci est définie par Avicenne comme la forme qui n'est pas la combinaison des qualités premières, mais une forme qui transcende la complexion dont elle est issue ${ }^{26}$. La notion de forme spécifique permettait ainsi de rendre compte de phénomènes physiques comme la vertu attractive de l'aimant sur le fer, ou de phénomènes médicaux comme la vertu attractive de l'humeur cholérique qui était prêtée à la rhubarbe ou encore celle de la thériaque, ce mélange complexe ayant des vertus médicinales très étendues. D'une certaine manière, la «forme spécifique " permettait d'expliquer ce qu'on ne pouvait pas expliquer; elle permettait de ne pas déroger à la chaîne explicative en encadrant par cette notion (en fait assez vide) le phénomène qui défiait le cadre explicatif dominant ${ }^{27}$.

On ne s'étonnera donc pas que la peste, qui se posa comme un défi pour les médecins, fût expliquée très souvent en recourant à cette notion. Ainsi, la corruption de l'air rendue responsable de l'épidémie était la plupart du temps considérée comme une altération substantielle de l'air ambiant: "nous croyons ", écrivent les médecins parisiens consultés en 1348, "que la présente épidémie ou peste provient d'un air corrompu en sa substance et non seulement altéré en sa qualité ${ }^{28}$. En d'autres termes, cette corruption de l'air correspond à une modification ayant trait à sa forme substantielle - la forme substantielle étant un peu ici un doublon de la forme spécifique ${ }^{29}$.

Dans les écrits sur la peste, on rencontre de nombreux cas de prescriptions de pierres précieuses : émeraudes, escarboucles etc. Dans la consultation parisienne de 1348 on lit que «l'émeraude est aussi un médicament remarquable contre tout poison $»^{30}$. Or l'action de l'air corrompu sur le corps était fréquemment assimilé à une forme d'empoisonnement. Sans doute faut-il faire déjà une distinction, attestée dans les écrits sur la peste eux-mêmes, entre les gemmes censées être utilisées par voie interne ( $a b$ intra) et celles qui doivent être utilisées par voie externe ( $a$ extra).

1. Les gemmes utilisées par voie interne se confondent en quelque sorte avec les médecines ordinaires agissant par leur forme spécifique. Ce sont, par exemple, des diamants réduits en poudre et destinés à être ingérés dans un mélange. Les occurrences sont très nombreuses dans la littérature des écrits sur la peste. Ainsi, dans nombre d'entre eux, l'émeraude est considérée, parmi les médecines cordiales, comme une 
"thériaque remarquable" contre tous les poisons ${ }^{31}$. On trouve, par exemple, dans le traité sur l'épidémie de Jean de Bourgogne ( $a u$ XIV siècle), après la description d'une recette d'un électuaire : «Si "le malade" est riche, on doit ajouter des perles, de l'or en "minces" feuilles, de l'argent pur, des hyacinthes, des émeraudes et de la corne de $\operatorname{cerf} »^{32}$.

On ne saurait mieux résumer la fonction d'ingrédients qui est assignée à ces gemmes, leur place particulière venant au fond de leur coût élevé qui en fait des médecines pour riches (une précision récurrente dans les écrits sur la peste). Leur action est expliquée comme une opération par la forme spécifique ou, comme on le trouve souvent formulée, a tota proprietate, par leur propriété tout entière. Que ce soit donc par le mode d'administration (la voie orale), par le mode d'action envisagé (la forme spécifique) ou par l'inscription dans le cadre étiologique de la peste (la préservation contre l'air empoisonné notamment), on peut dire que l'usage des pierres précieuses ab intra ne déroge pas vraiment à la méthode ordinaire de la médecine savante ${ }^{33}$.

2. Mais on franchit indéniablement un seuil quand on aborde l'utilisation ab extra des pierres précieuses. Dans son Regimen in pestilencia compilé en octobre 1378, après avoir présenté les six choses non naturelles dont il faut tenir compte pour se préserver de l'épidémie, le médecin Cardo de Milan aborde le regimen medicinarum, c'est-à-dire les médicaments à prendre par voie interne (interius sumendarum), puis ceux qui sont « approchés de l'extérieur » (exterius approximatis). Dans ces derniers, il aborde d'abord les parfums (odoramenta), puis en vient aux gemmes. C'est ainsi qu'il indique qu'un homme riche devra porter au doigt une escarboucle ou un rubis, puisque ces gemmes ont la vertu d'éloigner l'air et les vapeurs empoisonnées. Il renvoie plus généralement à d'autres pierres précieuses en se référant au De mineralibus (Des minéraux) d'Albert le Grand $^{34}$. Ce sentiment d'une différence entre l'utilisation $a b$ intra et ab extra n'est pas une impression de lecteur moderne, les auteurs médiévaux ont bien conscience eux aussi de se trouver, dans le second cas, devant un phénomène qui étonne, qui tient du merveilleux (comme, du reste, la vertu attractive de l'aimant); en effet l'action à distance dissimule (de façon pour ainsi dire immédiatement visible) les modalités de l'opération effectuée, ce qui est un des éléments de la définition des opérations magiques au sens large. Du reste, l'explication par la forme spécifique permet là encore d'intégrer le phénomène au cadre explicatif global. Un auteur aussi scrupuleux que Thomas d'Aquin ne paraît pas nier théoriquement le pouvoir de ce qu'on peut appeler les amulettes, c'est-à-dire de choses naturelles ou de parties de choses naturelles (pierres précieuses, membre d'animal, morceau de plantes...) ${ }^{35}$. En somme, l'utilisation d'amulettes dans les écrits sur la peste, est simplement plus merveilleuse dans sa manifestation que l'utilisation ab intra des gemmes. Elle non plus ne détruit pas l'étiologie du fléau élaborée par les médecins : il s'agit bel et bien de contrer les vapeurs et l'air empoisonnés rendus responsables de l'épidémie.

Dans un traité de 1405, dans le chapitre consacré aux apostumes (aux bubons), l'auteur signale qu'il ne s'étendra pas à détailler un grand nombre de médicaments étant donné l'urgence dans laquelle se trouvent les médecins. De plus, dans la mesure où les remèdes recherchés échouent souvent, on préférera les procédés communs, qui parmi les simples et les composés moins précieux évacuent la matière de manière sensible ou insensible. Or, après avoir évoqué les ventouses et les sangsues, le médecin décrit un singulier remède : 
$<$ Il faut prendre> un coq noir et vivant déplumé autour de l'anus et ayant l'anus fendu, il faut le mettre sur le bubon et le tenir là pendant un septième d'heure et ensuite l'enlever et mettre de nouveau un autre <coq> de la même manière à <cet> endroit, en continuant pendant toute une journée. En effet, le poison est attiré vers le cœur du coq s'il y a de la <substances nocive; voilà pourquoi le coq meurt immédiatement et aussitôt ${ }^{36}$.

La présence du coq noir et du chiffre sept a évidemment de quoi intriguer - sans parler du procédé lui-même, qui exigerait 168 coqs. Pourtant l'explication qui est donnée peut très bien mettre en jeu simplement la notion de propriété spécifique. Le corps du coq a la vertu d'attirer à lui le poison qui s'est fixé dans le bubon du pestiféré.

3. Évidemment un autre seuil est à nouveau franchi quand on aborde le domaine des opérations artificielles proprement magiques, telle la fabrication des talismans. On peut définir un talisman comme une chose artificielle ayant reçu une forme ou une figure et dont le pouvoir magique est censé advenir, au moins en partie, grâce à l'intervention artificielle du magicien. Thomas d'Aquin dénonce comme démoniaque tout talisman, c'est-à-dire toute figure artificielle utilisée dans une opération magique; Albert le Grand, lui, parvient à envisager l'existence d'un talisman naturaliste (qu'on appelle ailleurs «image astrologique » $)^{37}$. Trouve-t-on des talismans dans les traités sur la peste ? Jean-Noël Biraben a, en effet, rassemblé des témoignages d'usages populaires de talismans au temps du fléau ${ }^{38}$. Cette dérive talismanique a-t-elle aussi affecté les médecins eux-mêmes?

J'ai pu rassembler quatre mentions de sceaux astrologiques dans les écrits sur la peste. Les sceaux astrologiques sont une forme particulière de talismans astrologiques: ils représentent sur une médaille la constellation sous laquelle ils ont été fabriqués et sont censés avoir alors été dotés de vertu par l'influence de cette constellation et des autres configurations astrologiques. Outre une référence purement théorique chez Maino de Maineri, on trouve dans un traité de 1349 conservé à Erfurt un sceau du Lion prôné pour s'opposer directement à la cause céleste, et surtout deux références au sceau du Serpentaire pour se prémunir contre l'air empoisonné : chez Gentile da Foligno (1348) et Antonio Guaineri (av. 1440) ${ }^{39}$.

31 Le cas du Contra pestilentiam du médecin Gentile da Foligno est particulièrement significatif : il s'agit de l'un des tous premiers écrits sur la Peste noire. En se référant au De venenis de Pietro d'Abano, il explique que si l'on grave dans une hématite la figure de la constellation du Serpentaire (" un homme ayant le genou fléchi ceint d'un serpent, dont la main droite tient la tête et la main gauche la queue ») et que l'on monte cette pierre gravée sur un anneau d'or en insérant sous elle une racine de serpentaire, l'anneau ainsi constitué préservera celui qui le portera « de tout poison aussi bien de celui de l'air pestilentiel que de celui d'un minéral... ». Or une analyse précise permet de conclure que ce procédé évoqué au milieu de médecines reposant sur l'utilisation de la forme spécifique (comme la poudre d'émeraude) ne constitue pas, non plus, un cas de court-circuitage de la rationalité scolastique médicale. Gentile procède au contraire à une adaptation parfaitement méthodique d'un procédé qu'il tient d'une autorité médicale : Pietro d'Abano. Ce dernier avait repris à un texte traditionnel (le De lapidibus d'Azareus) l'empiricum de la figure du Serpentaire qui, gravée dans une hématite et portée dans un anneau d'or selon les conditions évoquées plus haut, devait lutter contre tous les poisons ${ }^{40}$. Or Gentile est confronté à une maladie (la peste) qui est interprétée comme un empoisonnement de l'air; il est donc logique d'utiliser ce procédé pour se prémunir de l'air empoisonné responsable de l'épidémie. Du point de 
vue de l'étiologie, du respect de l'autorité, et des règles déductives, Gentile en prônant l'utilisation du sceau du Serpentaire respecte en tout point le cadre rationnel de la médecine scolastique.

Mais d'une façon générale, l'utilisation de sceaux astrologiques est relativement rare dans les traités sur la peste. Si le champ médical a enregistré une inflexion significative vers l'occulte, c'est plutôt indirectement qu'il faut en attribuer une part de responsabilité à l'épidémie. En effet, à partir du xive siècle se multiplient les traités sur les poisons. Souvent dédiés à de hauts personnages, ils énumèrent les poisons, les antidotes et donnent d'autres conseils pour éviter les empoisonnements (comme le fait d'observer l'attitude du serviteur qui sert les mets à déguster). Il me semble que cette multiplication est à attribuer, en partie au moins, à l'épidémie de peste et à l'étiologie alors en vigueur qui en faisait un empoisonnement de l'air. La peste avait bel et bien amené l'obsession du poison, et la multiplication des écrits sur les poisons en témoigne. Or dans ces écrits sur les poisons, on trouve un grand nombre d'empirica merveilleux (davantage, en réalité, que dans les écrits sur la peste). Et pour des raisons liées aux sources (Azareus, Albert le Grand, Pietro d'Abano et Arnaud de Villeneuve) on trouve très fréquemment le sceau du Serpentaire ${ }^{41}$.

Quoi qu'il en soit, paradoxalement, loin de témoigner d'une tentation de l'irrationnel, la présence des éléments magiques que l'on trouve dans les écrits sur la peste attestent au contraire la vigueur persistante de l'aptitude des médecins à trouver des solutions rationnelles face au fléau en restant dans le cadre rigoureux de leur discipline. Sans doute, que ce fût à travers la croissance relative de la part de l'astrologie ou de celle des empirica, ou bien encore à travers l'essor concomitant des traités sur les poisons qui prônaient en abondance les procédés empiriques plus ou moins merveilleux, la peste contribua-t-elle pour une part à fissurer l'édifice de la médecine scolastique; mais, là comme dans le domaine démographique, la peste joua plus un rôle de catalyseur que de déclencheur de phénomènes.

Face au défi que représenta la peste, qu'il s'agît de proposer une étiologie où intervenaient les astres ou de prescrire des moyens de s'en préserver ou de la guérir, y compris avec des empirica merveilleux, les médecins de la fin du Moyen Âge surent raison garder.

\section{NOTES}

1. D. JACQUART, «La scolastique médicale », dans M. D. GRMEK dir., Histoire de la pensée médicale en Occident. 1. Antiquité et Moyen Âge, Paris, 1995, p. 175-210 ; N. WEILL-PAROT « Médecine et ésotérisme [Moyen Âge] ", dans Dictionnaire critique de l'ésotérisme, J. SERVIER dir., Paris, 1998, p. 825-829.

2. D. JACQUART, « Médecine et astrologie à Paris dans la première moitié du XIV siècle ", dans G. FEDERICI VESCOVINI et F. BAROCELLI éd., Filosofia, scienza e astrologia nel Trecento europeo, Padoue, 1992, p.121-134 ; EAD., « Theory, Everyday Practice and Three Fifteenth-Century Physicians », Osiris, n.s., 6, 1990, p. 140-160, réimpr. dans EAD., La 
science médicale occidentale entre deux renaissances, $\mathrm{XII}^{e}-\mathrm{XV}^{e}$ s, Aldershot, 1997 ; EAD., «L'influence des astres sur le corps humain chez Pietro d'Abano », dans B. RIBÉMONT éd., Le Corps et ses énigmes au Moyen Âge, Caen, 1993, p. 73-86 ; EAD., « Die Rationalisierung des Menschen und der Welt in der Medizin des Mittelalters », dans K. FLASCH et J. R. JECK éd., Das Licht der Vernunft. Die Anfänge der Aufklärung im Mittelalter, Munich, 1997, p. 84-99 ; EAD., La médecine médiévale dans le cadre parisien, Paris, 1998, p. 448-465.

3. G. FEDERICI VesCOVINI, « Peter of Abano and Astrology », dans P. CURRY éd., Astrology, Science and Society : Historical Essays, Woodbridge, 1987 ; p. 19-39 ; D. JACQUART, "L'influence des astres... », loc. cit.

4. A. M. CAMPBELL, The Black Death and Men of Learning, New York, 1931 ; J.-N. BIRABEN, Les Hommes et la peste en France et dans les pays européens et méditerranéens, 2 vol., Paris-La Haye, 1975 ; K. BERGDOLT, Der Schwarze Tod in Europa. Die grosse Peste und das Ende des Mittelalters, Munich, 1994 ; J. ARRIZABAlaGA, « Facing the Black Death : Perceptions and Reactions of University Medical Practitioners ", dans L. GARCÍA-BALLESTER, R. FRENCH, J. Arrizabalaga et A. Cunningham éd., Practical Medicine from Salerno to the Black Death, New York, 1994, p. 237-288 ; D. JACQUART, « Die Rationalisierung... », loc. cit. ; EAD., La Médecine médiévale dans le cadre parisien..., p. 230-265.

5. E. Delaruelle, La Piété populaire au Moyen Âge, Turin, 1975, p. 277-327 ; J. DelumEAu, La Peur en Occident (XIV ${ }^{e}$-XVIII ${ }^{e}$ siècles). Une cité assiégée, Paris, 1978, p. 98-142 ; M. VoVELLE, La Mort en Occident de 1300 à nos jours, Paris, 1983, p. 89-167 etc.

6. C. CRISCIANI, «Alchemy and Medicine in the Middle Ages : Recent Studies and Projects of Research », Bulletin de philosophie médiévale, 38, 1996, p. 9-21; EAD., "Oro potabile fra alchimia e medicina : due testi in tempo di peste ", dans F. CALASCIBETTA éd., Storia e fondamenti della chimica (Atti del VII Convegno Nazionale, L'Aquila, 8-11 ottobre 1997), Memorie di Scienze Fisiche e Naturali, vol. 115, « Rendiconti della Accademia Nazionale delle Scienze detta dei XL », serie V, vol. XXI, parte II, t. II, 1997, p.83-93 ; C. CRISCIANI, M. PEREIRA, « Black Death and Golden Remedies. Some Remarks on Alchemy and the Plague ", dans A. PARAVICINI BAGLIANI et F. SANTI éd., The Regulation of Evil. Social and Cultural Attitudes to Epidemics in the Late Middle Ages, Florence, 1998, p. 7-39 (Micrologus' Library, 2).

7. K. SudHOFF, «Pestschriften aus den ersten 150 Jahren nach der Epidemie des "Schwartzen Todes" ", Archiv für Geschichte der Medizin (= AGM), 2-17, 1909-1925.

8. J. ARRIZABAlAGA, « Facing the Black Death... », loc. cit.

9. Éd. dans E. H. REBOUIS, Étude historique et critique sur la peste, Paris, 1888, p. 70-145 ; voir D. JACQUART, La Médecine médiévale..., op. cit., p. 230 et suiv. ; cf. ALBERT LE GRAND, De causis proprietatum elementorum, II.2.1, P. HossfELD éd., dans Alberti Magni Opera omnia, V/II, Münster, p. 96, 1. 53-68.

10. JACME D'AGRAMONT, Regiment de preservacio a epidemia o pestilencia e mortaldats, I. 1. 2, J. Veny i Clar éd., Tarragona, 1971 ; M.-L. Duran-Reynals et C. E. A. Winslow, « Regiment de Preservacio a Epidimia o Pestilencia e Mortaldats. Epistola de Maestre Jacme d'Agramont... Translated in English ", Bulletin of the History of Medicine, 23, 1949, p. 57-89 (p. 66).

11. E. H. ReBouis éd., Étude historique..., op. cit., p. 72.

12. La Tête du Dragon est le point ascendant de l'intersection que fait l'orbite de la lune avec le plan de l'écliptique (J.-P. BOUDET, Le Recueil des plus célèbres astrologues de Simon de Phares, II, Paris, 1999, Annexe II, p. 372). 
13. Une planète est dite rétrograde quand, à un certain moment de sa révolution, son mouvement apparent va dans le sens inverse de l'ordre du Soleil et des signes du zodiaque, ibid., p. 386-387.

14. À cet égard, un astrologue aussi soucieux de rester dans le cadre naturaliste que l'était l'anglais John of Eschenden ne manqua pas d'évoquer au nombre des phénomènes naturels de grande ampleur provoqués par les astres, précisément la peste de 1348 mise en relation avec la conjonction de 1345. Sur cet astrologue, voir H.M. CAREY, Courting Disaster. Astrology at the English Court and University in the Later Middle Ages, Londres, 1992.

15. Sur l'analyse originale de la peste que livre le naturaliste Conrad de Mengenberg dans son Tractatus de mortalitate in Alamannia et, en particulier, la place qu'il assigne ou n'assigne pas aux astres, voir J. PfEIFfER, « Macht der Stern oder Miasmen der Erde. Heinrich von Mügeln und Konrad von Megenberg über die Pest von 1348 ", dans U. SCHAEFER, Artes im Mittelalter, Berlin, 1999, p. 110-123, en particulier p. 118-123. Dans son raisonnement complexe, Conrad écrit : « non apparet michi hanc mortalitatem immediate fore ab astris, sed pocius mediate aut multum remote » (éd. par S. KRÜGER, « Krise der Zeit als Ursache der Pest ? ", dans Festschrift für Hermann Heimpel. Zum 70. Geburstag am 19. September 1971, Mitarbeiten des Max-Planck-Instituts für Geschichte éd., Göttingen, 1972, p. 839-883, p. 870).

16. K. SUdhoff éd., « Pestschiften... », AGM, 16, 1925, nº 177, p. $20-29$ (p. 22) : « Deus et celum per se non sunt causa epydimie. [...] De celo patet, quia sic in omni loco et in omni tempore et in omni homine equaliter esset pestilencia, cum celum vndique equaliter se habet vnde a quarto Canone de febre pestilenciali causa prima longiqua ad fe. pestilencialem sunt figure celestes et propinque dispositiones terrestres. Deus est causa epydimie remotissima, remocior celum, aer remotus, humor propinquus, aer putridus propinquior, vapor putridus in corde infusus propinquissima ». Johann (ou Jean) von Sachsen vivait encore en 1409, voir E. WICKHERSheimer, Dictionnaire biographique des médecins en France au Moyen Âge, Genève, 1936, rééd. 1979, II, p. 475. Il ne doit pas être confondu avec l'astronome de la première moitié $\mathrm{du} \mathrm{Xv}^{\mathrm{e}}$ siècle, Jean de Saxe, voir E. PoulLE, «John of Saxony », dans Dictionary of Scientific Biography, VII, 1973, p. 139-141 (p. 140).

17. K. SudHoff éd., « Pestschiften... », AGM, 17, 1925, n. 265, p. 77-92 : « ... multi sapientes causam istius terroris pestilencie nescierunt attribuere nisi soli deo, tamen causari inmediate a constellatione sive a celo mediante vapore corrupto probabile est, sicut causatur radius a sole, et ista videtur esse astrologorum intencio, qui dicunt unam planetam esse benivolum et alium malivolum, sed pluri medici hoc non considerant ». Le traité est conservé dans un manuscrit composite (Hannovre, Niedersächsische Landesbibliothek, IV.339, $\mathrm{f}^{\circ} 171 \mathrm{r}^{\circ}$ et suiv.) comprenant des textes alchimiques, astrologiques et médicaux ; le texte de Primus se trouve inséré dans une série de textes médicaux, $c f$. Н. НÄRTEL et F. ЕкоWSкI, Handschriften der niedersächsischen Landesbibliothek Hannover, 2 vol., Wiesbaden 1982-1989, II, p. 121-127. E. WICKHERSHEIMER, Dictionnaire biographique..., op. cit., p. 670, conteste l'hypothèse de K. Sudhoff qui faisait de ce médecin, qui se qualifie lui-même de « Parisius, in artibus et medicina professor », un natif de Goerlitz, alors que ce dernier parle de la Sicile comme de sa patrie natale. 18. Giovanni Santasofia, fils et père de médecins, étudie à Padoue puis à Pérouse, devient médecin de Louis II de Gonzague à Mantoue, puis, peu avant 1375, revient enseigner à Padoue, voir T. PESENTI, « Il proemio del commento di Giovanni Santasofia 
alla Tegni di Galeno ", Quaderni per la Storia dell'Università di Padova, 33, 2000, p. 27-44. K. Sudhoff éd., « Pestschiften... », AGM, 6, 1912, n. 50, p. 344-349, ici p. 344-345, 1. 1-14 : «Venerandi domini, haec pestis tam admirabilis et horrenda a causis superioribus procedat regulantibus et gubernantibus corpora nostra, quorum influxus prohibere non possumus ".

19. Ibid., p. 345, 1. 5-11 : «Quia tamen secundum Galenum primo de febribus maxima particula generacionis aegritudinum est passim corporis praeparatio, aliqua remedia scribam a nostris autoribus aprobata et in aliis pestibus experimentata, quae etsi superiorum influxus non prohibent, corpora sic nostra disponunt, ut non de levi a praedictis superioribus causis patiantur ».

20. D. JACQUART, « La scolastique médicale », loc. cit., p. 205-206.

21. Conrad de Megenberg rejette l'accusation portée contre les juifs et passe à l'analyse de l'explication astrologique jugée « magis philosophica », éd. par S. KRÜGER, "Krise der Zeit... », loc. cit., p. 866-868. Sur la position de Conrad face à cette rumeur, voir aussi S. GUERCHBERG, «Les controverses sur les prétendus semeurs de la Peste Noire d'après les traités de peste de l'époque ", Revue des études juives, n. s., 8, 1948, p. 3-40.

22. GUY DE CHAULIAC, Inventarium sive Chirurgia magna, II. 2. 5, M.S. OGDEN et M. McVAUGH éd., 2 vol., Leyde, 1997, vol. II, p. 118, 1. 22-30.

23. K. SudHoff éd., « Pestschriften... », AGM, 17, 1925, n. 265, p. 77-92, p. 79, 1. 103-110 : « Tercium est quod iudei minus moriuntur quam christiani. Respondetur quo $<\mathrm{d}>$ proprio victu judeorum [correction proposée par K. Sudhoff au lieu de christianorum] non se exponunt aeri mane nec sero nec ita sepe proambulant ad aerem exteriorem, qui est peyor in tempore pestilenciali, quando stelle habent malas influencias. Posset dici, quod iudei sunt saturnini, cuius planete compleccio est frigida et sicca, opposita morbo pestilenciali, et sic plus resistere et minus a tali aere infecto passibiles et sic iudei minus moriuntur $"$.

24. Séraphine Guerchberg s'était demandé dans quelle mesure, en continuant d'accréditer ce fait, Primus de Görlitz se mettait « sciemment » ou non " au service de l'antisémitisme ", le fait que cette opinion fût alors considérée comme établie étant un indice du renforcement l'hostilité à l'égard des juifs au XV $\mathrm{X}^{\mathrm{e}}$ siècle (S. GUERCHBERG, « Les Controverses... ", loc. cit., p. 38).

25. Voir note 1.

26. Avicenne, Canon, Liber I, Fen II, Doctrina II, Summa I, cap. 15, Venise, 1505, fo $30 \mathrm{r}^{\circ} \mathrm{a}$. Sur la forme spécifique, voir par ex., B. P. COPENHAVER, « Scholastic Philosophy and Renaissance Magic in the De vita of Marsilio Ficino », Renaissance Quarterly, 37, 1984, p. 523-554.

27. N. WEILL-PAROT, « Encadrement ou dévoilement : l'occulte et le secret dans la nature chez Albert le Grand et Roger Bacon », Micrologus (à paraître).

28. I. 2, E. H. ReBouIS éd., Étude historique..., op. cit., p. .

29. Voir J. ARRIZABALAGA, « Facing the Black Death... », loc. cit., p. 259-264 ; sur l'identification possible ou non de la forme substantielle avec la forme spécifique, voir M. R. McVAUGH, «The Development of Medieval Pharmaceutical Theory »= introduction à ARNALDI DE VILLANOVA, Opera medica omnia, II. Aphorismi de gradibus, M. R. McVAugh éd., Grenade-Barcelone, 1975, p. 84, p. 19 note 11 ; B. P. COPENHAVER, « Scholastic Philosophy... », loc. cit., p. 541 et note 48 ; E. KARGER, « Richard Rufus's Account of Substantial Transmutation ", Medioevo, 27, 2002, p. 165-189 (je remercie Irene Caiazzo de m'avoir indiqué cet article éclairant) ; T. DAGRON, « La doctrine des 
qualités occultes dans le De incantationibus de Pomponazzi », Revue de métaphysique et de morale (à paraitre : je remercie l'auteur de m'avoir offert la possibilité de lire cet article avant sa parution).

30. II. 2, E. H. ReBouIs éd., Étude historique..., op. cit., p. 126 : « Smaragdus est etiam insignis medicina contra omne venenum. »

31. Regimen praeservativum a pestilencia ex purificacione aeris, AGM, 11/1-2, 1918, $\mathrm{n}^{\circ} 110$, p. 66, 1.285 : «Smaragdus inter omnes medicinas cordiales est insignis tyriaca contra omne venenum »; Compendium epidemiae, AGM, 11/3-4, 1919, $\mathrm{n}^{\circ}$ 122, 1. 385 : « Smaragdus insignis tiriace contra omne venenum est. »

32. K. SUDHoff éd., « Pestschriften... », AGM, 5, 1912, $\mathrm{n}^{\circ}$ 27, p. 77-80, 1.214 : « Si dives fuerit, debent poni cum hiis margaritae, aurum foliatum, argentum purum, iacincti, smaragdi et os de cornu cervi [plutôt que cordecervi]». Sur les problèmes liés à l'identité de ce personnage (en particulier les débats autour de son hypothétique identification avec Jean de Mandeville) : E. WICKERSHEIMER, Dictionnaire, op. cit., I, p. 369-370 ; D. JACQUART, Supplément [au Dictionnaire... de Wickersheimer], Genève, 1979, p. $147-148$.

33. Je suis reconnaissant à Mme $\mathrm{D}$. Jacquart d'avoir attiré mon attention sur ce point il y a quelques années lorsque je préparais l'article « Médecine » pour le Dictionnaire critique de l'ésotérisme.

34. K. SudHoff éd., « Pestschriften... », loc. cit., AGM, 6, 1913, nº 45 [Munich, Bayerishe Staatsbibliothek, lat. 323, XIV siècle], p. 317-328, p. 325, 1. 352-357 : « Item de carbunculo et rubino. Similiter quilibet magnificus et potens debet portare in digito carbunculum vel rubinum, unum aut plures, quoniam aerem venenosum et vapores fugat. Et aliorum omnium lapidum pretiosorum virtutes dignoscuntur habere secundum venerabilem Albertum De mineralibus », cf. ALBERT LE GRAND, De mineralibus, II.II.3.

35. THOMAS D'Aquin, Summa theologiae, II.II. q. 96, art. 2, ad 1 ; B. P. CoPenHAVER, "Scholastic Philosophy... », loc. cit., p. 532.

36. K. SuDHoff éd., « Pestschriften... », loc. cit., AGM, 11/3-4, 1919, $\mathrm{n}^{\text {os }} 114-115$ (Tractatus de peste sananda ad rectores Erfordienses, rédigé à Erfurt en 1405, conservé dans le manuscrit Wolfenbüttel, Herzoglichen Bibliothek Helmstedter $783, \mathrm{f}^{\circ} 13 \mathrm{r}^{\circ}-24 \mathrm{v}^{\circ}$ ), p. 75-92, p. 85-86, 1. 410-415: « Tercium est gallus niger vivus circa anum deplumatus et ano ipso fisso poni expedit supra tumorem et ibi teneri per septimam partem unius hore et tunc removeri et alius loco ipsius iterum eodem modo reponi continuendo per unam totam diem ; attrahitur enim venenum ad cor galli, si assit nocivum, ideoque gallus subito et statim moritur. » Sur un procédé voisin, l'utilisation comme cataplasmes d'escargots vivants appliqués sur les bubons, voir D. LE BLÉVEC, « Un Médecin vivarois à la cour pontificale d'Avignon : Raimond Chalmel », Revue du Vivarais, 90, 1986, p. 13-24, p. 24 (je remercie l'auteur de m'avoir signalé ce dernier procédé).

37. N. WeILL-PAROT, Les «Images astrologiques » en Occident. Spéculations intellectuelles et pratiques magiques ( $\mathrm{XII}^{e}-\mathrm{XV}^{e}$ siècle), Paris, 2002, p. 223-302. (Pour une définition générale des talismans, v. notamment B. COPENHAVER, loc. cit.)

38. J.-N. BIRABEN, Les Hommes et la peste..., op. cit., II, p. 58-62.

39. N. WeILL-PAROT, Les « images astrologiques »..., op. cit., p. 591-570 : R. SimONINI éd., Maino de Maineri e il suo Libellus de preservatione ab epydimia, Modène, 1923, p. 15 ; Quaeritur primo quae sint egritudines nunc currentes..., K. SUDHOFF éd., AGM, 11, 1918, $\mathrm{n}^{\circ}$ 107, p. 51-44 ; GenTILE DA Foligno, Contra Pestilentiam, s.l.n.d. [Pellechet, $\mathrm{n}^{\circ}$ 5021], b 2v. ; 
ANTONIO GuAINERI, De peste, II.2.2, Venise, 1517, fo $105 \mathrm{v}^{\circ} \mathrm{a}$. (A. M. CAMPBELL, The Black Death..., op. cit., p. 16 et 71, avait déjà relevé les talismans dans le deuxième et le troisième de ces écrits).

40. Gentile da Foligno, Contra Pestilentiam, op. cit., fo b 3 v. [= p. 22] : « Nona medicina optima habita in Libro Persarum regum, et auctoritate Conciliatoris est quod si inculpiri feceris in lapide emathitis virum genuflexum cinctum serpentem cuius caput teneat dextera manus et caudam sinistra, et posueris lapidem hunc in anulo aureo, et sub lapide posueris radicem serpentarie tecumque portaveris hunc anulum, ab omni veneno te preservat tam aeris pestilentialis quam alterius minere... ». Cf. PIETRO D'ABANO, De venenis, cap. 4, dans ID., Conciliator, Venise, $1565, \mathrm{f}^{\circ} 264 \mathrm{v}^{\circ} \mathrm{b}-265 \mathrm{r}^{\circ} \mathrm{a}$ : « Aliud est etiam quod scriptum est in libro regum Persarum, quod si sculpire feceris in lapide haematitis virum genuflexum cinctum serpente, cuius caput teneat dextra manus et cauda sinistra, posuerisque hunc in aureo annulo, et sub lapide posueris radicem serpentariae tritam, portaverisque hunc annulum, ab omni veneno praeservat. Quod quidem ego quandoque praeparari feci, et ad praedictum usum servari. » Ce « Livre des rois de Perse » correspond au De lapidibus attribué à Azareus, comme je l'ai montré : N. WEILLPAROT, Les « Images astrologiques »..., op. cit., p. 115-116 et 529-530.

41. N. WEILL-PAROT, Les «Images astrologiques »..., op. cit., p. 575-588. Sur la question politique et juridique des empoisonnements, voir F. COLLARD, Le Crime de poison au Moyen Âge, Paris, 2003.

\section{RÉSUMÉS}

Confrontée à la nouveauté que constitua l'arrivée de la peste en Occident, la médecine savante fut apparemment mise à rude épreuve. Son inadéquation n'était-elle pas visible face à ce défi pratique? Pourtant, l'étiologie astrologique du fléau resta assez strictement naturaliste (la grande conjonction de 1345 fut considérée comme la cause de la putréfaction de l'air tenue ellemême pour responsable de la Peste Noire de 1348). Quant au recours aux empirica merveilleux ou magiques, il fut relativement limité et toujours encadré par une logique d'application respectueuse de la théorie médicale : qu'il s'agisse des gemmes ingérées comme des médicaments ou bien portées en amulettes, ou même des sceaux astrologiques talismaniques.

The medical rationality faced with the plague: medicine, astrology and magic (1348-1500). Facing the novelty created by the advent of the Plague in Western Europe, learned medicine was apparently put to the test. Was not obvious its inadequacy to the practical challenge ? Yet, the astrological aetiology of the scourge remained quite strictly naturalistic (the great conjunction occuring in 1345 being the cause of the putrefaction of the air responsible for the Black Death in 1348). Concerning the use of marvelous or magical empirica, it was rather limited and always framed by a logical and respectful applying of the medical theory, as shown by the gems ingested as medicines or carried as amulets, or even astrological-talismanic seals. 
INDEX

Mots-clés : magie, médecine savante, peste, rationalité, astrologie

Keywords : magic, learned medicine, plague, rationality, astrology

\section{AUTEUR}

\section{NICOLAS WEILL-PAROT}

Université Paris-VIII, Département d'Histoire, 2 rue de la Liberté, F-93526 Saint-Denis cedex 02 